\title{
Glottopol
}

Revue de sociolinguistique en ligne

$34 \mid 2020$

Les « langues de France ", 20 ans après

\section{L'épervier, la cage et le passereau}

\section{Pascal Ottavi}

\section{OpenEdition}

Journals

Édition électronique

URL : https://journals.openedition.org/glottopol/501

DOI : 10.4000/glottopol.501

ISSN : 1769-7425

\section{Éditeur}

Presses universitaires de Rouen et du Havre

\section{Référence électronique}

Pascal Ottavi, «L'épervier, la cage et le passereau », Glottopol [En ligne], 34 | 2020, mis en ligne le 21 juillet 2020, consulté le 02 octobre 2021. URL : http://journals.openedition.org/glottopol/501 ; DOI : https://doi.org/10.4000/glottopol.501 


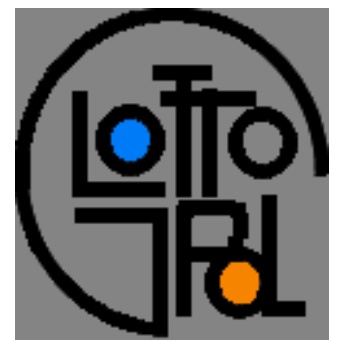

\section{GLOTTOPOL}

Revue de sociolinguistique en ligne $\mathrm{n}^{\circ} 34$ - juillet 2020

Les "langues de France " : 20 ans après

\section{SOMMAIRE}

Hommages à Jean Le Dî

Christian Lagarde : «Langues de France ». Au-delà du symbolique?

Entretien avec Bernard Cerquiglini, par Christian Lagarde.

Entretien avec Paul de Sinety, Délégué général à la langue française et aux langues de France, par Christian Lagarde.

Georg Kremnitz : La problématique initiale de la liste Cerquiglini et ses effets ultérieurs.

Alain Viaut : De "langue régionale » à " langue de France » ou les ombres du territoire.

Wanda Mastor: Le statut constitutionnel des langues régionales en droit comparé. De la reconnaissance à l'indifférence.

Philippe Martel, Marie-Jeanne Verny: Les langues régionales au Parlement, ou l'éternel retour.

Romain Colonna : Les "langues de France » : des langues non-étatiques au pays de l'Étatnation.

Hervé le Bihan : La langue bretonne : une visibilité toute en retenue.

Véronique Bertile : Les langues d'outre-mer: des langues de France? Approche juridique.

Jacques Vernaudon: Les langues polynésiennes et kanak, des "langues de France » en contexte de décolonisation.

Luc Biichlé : Qu'advient-il de l'arabe de France? Mise en perspective sociolinguistique...

Pascal Ottavi : L'épervier, la cage et le passereau.

Marielle Rispail : Le francique lorrain, langue de France? Réflexions et témoignages.

\section{Compte rendu de lecture}

Par Salih Akin : Jean Le Dû \& Yves Le Berre, Métamorphoses. Trente ans de sociolinguistique à Brest (1984-2014), Brest, Centre de Recherche Bretonne, 2019, 302 p. 


\section{L'ÉPERVIER, LA CAGE ET LE PASSEREAU}

\section{Pascal Ottavi Università di Corsica Pasquale Paoli, Corte, UMR CNRS 6240 LISA}

Si la métaphore peut trouver place de façon plus ou moins discrète dans un texte universitaire, le recours à la parabole n'y parait guère d'usage. On connait pourtant, en anthropologie, celle du bouc-émissaire, magistralement illustrée par René Girard dans son analyse de l'origine de la violence dans la nature humaine (1972). En littérature, Jérôme Ferrari, avec son roman Sermon sur la chute de Rome ${ }^{1}$, en use avec talent, notamment lors du récit final de la mort de Saint Augustin à Hippone. Marcu Biancarelli, s'exprimant cette fois-ci en corse, narre dans l'une de ses nouvelles, U pulpu, l'argusta è a murena ${ }^{2}$, le jeu complexe des trois protagonistes marins lorsqu'ils se trouvent pris dans la même nasse: en milieu aquatique chacun constitue un maillon adéquat de la chaine alimentaire, le poulpe se livre à la prédation de la langouste tandis que cette dernière s'en prend à la murène, laquelle ne dédaigne pas le chasseur initial. Dans le cas d'espèce, les trois animaux se trouvent pris au piège car voici ce qui va se passer : «...le poulpe, la langouste et la murène vont mourir d'inanité mais personne ne mangera personne, personne n'attaquera sa proie, parce que le premier qui attaque prête le flanc à l'agression de son prédateur. Ainsi, au même endroit, dans le même temps, tous les agresseurs sont aussi devenus des proies $»^{3}$.

Ne possédant pas le talent d'écriture des trois auteurs cités, l'anthropologie et la littérature ne constituant pas l'objet de son propos, l'auteur du présent texte s'est permis d'inventer pour l'occasion la parabole de l'épervier, de la cage et du passereau à partir d'une anecdote qui lui a été narrée, toujours en corse, voici plus de quarante ans, par un vieil homme profondément marqué par la ruralité. Ayant capturé un merle, il le plaça dans une cage ; il nourrissait le petit volatile et trouvait plaisir à entendre son chant, et ce malgré le dicton «L'ocellu in gabbia, ùn canta d'amore chi canta di rabbia $»{ }^{4}$. Passionné de chasse, il captura quelques temps après, sans le blesser, un épervier, qu'il introduisit lui aussi dans la même cage, celle-ci étant suffisamment grande pour accueillir deux hôtes à la fois. Le lendemain, il découvrit que, du passereau noir au chant mélodieux, ne demeuraient plus que les plumes. L'épervier, lui, paraissait rassasié... Notre homme étant plus amateur des faits de nature qu'ornithologue, on comprend aisément le résultat inéluctable de l'interaction entre le prédateur et sa proie...5.

\footnotetext{
1 2012, Arles, Actes Sud.

2 « Le poulpe, la langouste et la murène », in Stremu meridianu, 2007, Aiacciu, Albiana.

${ }^{3}$ p. 77 , traduit par nous-même.

${ }^{4}$ « L'oiseau en cage ne chante pas par amour mais mû par la rage ».

5 «L'épervier d'Europe (Accipiter nisus) est un petit rapace commun et en expansion. Il se nourrit presque exclusivement d'oiseaux (mésanges, pinsons, grives, merles, étourneaux...). Le mâle étant d'une taille inférieure
} 
La parabole dont il est ici question met en présence trois actants : l'État a fabriqué la cage, l'épervier incarne l'idéologie républicaine, ou plutôt républicaniste, tandis que les langues régionales endossent le plumage sombre du fragile passereau... Ainsi l'étude ici commise se veut une lecture - provisoire, espérons-le - d'un processus de prédation linguistique, de « glottophagie » (Calvet 1979) voulu, institué et assumé apparemment sans état d'âme dans le cadre de la réforme du baccalauréat présentée en 2019, à 1'Assemblée nationale et au Sénat, par l'actuel ministre de l'Éducation. C'est en tout cas la lecture que l'auteur de ces lignes fait des bouleversements majeurs introduits dans le diplôme consacrant la fin des études secondaires et offrant, en partage désormais avec le dispositif parcoursup, le sésame d'entrée à l'université. D'ailleurs si l'on en croit Patrick Sauzet (1988), ce qui advient était en fait déjà écrit, comme on pourra le découvrir plus avant.

Pour étayer sa démonstration et sans revenir aux temps du déluge, il procèdera tout d'abord à l'analyse d'un texte capital : la circulaire de Monzie, produite en 1925 ; il reviendra ensuite, mais sans s'appesantir sur une matière bien maitrisée aujourd'hui, sur les principaux textes marquant un changement de cap positif, ceci jusqu'à l'actuelle réforme du baccalauréat. Le texte fondamental du ministre de l'instruction publique, celui qui mit sous l'éteignoir, pour plus de trente ans, la question de ce l'on n'appelait pas encore les langues régionales, offre en outre l'avantage d'exposer des prémisses qui font non seulement écho aux discours de Barère et de Grégoire mais qui instillent également leur présence discrète dans des textes et chez des auteurs contemporains, on veut parler ici du rapport Cerquiglini $(1999)^{6}$ ou encore du positionnement du philosophe Vincent Descombes, dont la lecture offre matière à réflexion.

Ce que l'on entend mettre ici en lumière, c'est que l'État, en France, a fait consciemment un choix sacrificiel à l'égard de toutes les langues autres que le français, que cette volonté s'est traduite non seulement dans les décisions institutionnelles, dans l'érection subséquente d'une mentalité dépréciative à l'égard des "patois » mais aussi qu'elle procède de justifications théoriques qui fondent et inspirent, non point forcément directement mais, quoi qu'il en soit, inéluctablement, un objectif et une stratégie d'unilinguisme sans appel.

\section{Une proposition initiale qui déstabilise l'enseignement d'une discipline au lycée}

Mais qu'est-ce qui a bien pu déclencher une déstabilisation de l'offre d'enseignement des langues régionales alors que, depuis quasiment soixante-dix ans, un certain modus vivendi s'était installé, permettant aux élèves de toutes les régions concernées d'accéder à un enseignement dont les conditions étaient certes perfectibles mais qui offrait au moins une certaine stabilité ?

C'est bien le nouveau bac qui remet malheureusement tout en question: la baisse d'attractivité des épreuves en constitue la preuve manifeste et le risque majeur. Peut-être n'y at-il pas de volonté consciente, au départ, de provoquer de telles conséquences, du moins veuton l'espérer'? ? L'ambition, si l'on ose dire, procède essentiellement d'une logique économique : alléger substantiellement le volume des dépenses obligatoires, en cohérence avec l'objectif fixé par l'État de supprimer 120000 postes de fonctionnaires sur le quinquennat. Les langues régionales et les enseignements quantitativement mineurs en termes de démographie lycéenne deviennent de fait les victimes collatérales des économies d'échelle attendues. Avec

à celle de sa "compagne”, il s'attaque à des proies plus petites (jusqu'à 120 grammes), la femelle pouvant elle tuer des oiseaux plus gros qu'elle, comme le pigeon ramier ». Source: https://www.ornithomedia.com/pratique /conseils/predation-epervier-europe-oiseaux-jardins-que-peut-faire-01060/.

${ }^{6} \mathrm{https}: / / \mathrm{www} . v i e-p u b l i q u e . f r / s i t e s / d e f a u l t / f i l e s / \mathrm{rapport} / \mathrm{pdf} / 994000719 . \mathrm{pdf}$.

7 https://www.alternatives-economiques.fr/enjeux-caches-de-reforme-bac/00083206. 
le piège redoutable de laisser croire que leur disparition tiendra avant tout au seul choix des familles.

Une fois pris connaissance des nouveaux contenus du bac, lesquels ont tardé à être divulgués, les premiers constats sont apparus sans appel :

- Le nombre d'opportunités pour le/la futur(e) candidat(e) est réduit : suppression de la LV2 approfondie, de la LV3 de spécialité, de la possibilité de choisir une deuxième option facultative.

- L'option facultative est dévalorisée : dans le cadre d'un choix exclusif (le candidat ne peut en prendre qu'une et une seule), celle-ci se voit complètement démonétisée avec la prise en compte des seuls points au-dessus de la moyenne, soit environ $1 \%$ de la note finale.

- L'option facultative "bonifiante ", si elle n'a plus cours pour les LR, est par contre maintenue pour les langues anciennes avec un coefficient bien plus attractif (multiplication des points au-dessus de la moyenne par 3, possibilité de seconde option dans le seul cas du choix de celles-ci).

- Le parcours d'excellence s'appuyant sur l'offre de LV2 approfondie pour atteindre le niveau B2 du Cadre européen commun de référence des langues se voit remplacé par l'enseignement de spécialité de langue régionale. Si celui-ci bénéficie d'un nombre $d$ 'heures de cours conséquent ainsi que d'un coefficient très important $(16 \%)$, il est impossible de le coupler par exemple avec l'enseignement de spécialité d'une autre langue puisque toutes sont regroupées sous l'intitulé « langues étrangères et régionales ». De plus, un tel choix aura des conséquences quasi définitives quant à la stratégie personnelle du lycéen vis-à-vis de la procédure parcoursup puisqu'il aura lui-même créé, dans une période de l'adolescence où psychologiquement les incertitudes sont bien plus grandes que les assurances quant à l'avenir, les conditions d'un choix plus restrictif quant à la proposition de poursuite d'études par les établissements d'enseignement supérieur.

- Il n'est plus possible de se présenter en candidat libre, ce qui exclut tous les candidats potentiels issus de lycées dans lesquels il n'y a pas d'enseignement de langue régionale. Si cette dernière occurrence affecte particulièrement les langues de l'Hexagone, c'est évidemment moins le cas pour le corse puisque tous les établissements, qu'ils soient d'enseignement général et technologique ou professionnel, offrent cette opportunité.

\section{La circulaire de Monzie : clôture ou relégation ontologique ?}

Abordons donc l'examen de la circulaire du 14 août $1925^{8}$, signée du ministre de l'Instruction publique Anatole de Monzie. Nous ne le ferons ici que de façon très synthétique : pour une analyse plus exhaustive, on voudra bien se reporter à Ottavi (2008). Ce texte vise à mettre un terme aux débats qui traversent à l'époque le corps politique et le corps enseignant quant à la place éventuelle à réserver aux langues locales dans l'école publique. Les lois Ferry avaient (enfin) offert, quasiment un siècle après la révolution, les moyens d'une politique linguistique radicale. Qui plus est, le soutien contemporain de l'Église à certains dialectes (le breton notamment, usé dans les sermons) est exploité comme argument d'expansion sans appel de la langue de la laïcité (Chervel 1995). L'examen de la circulaire va permettre de mieux apprécier la césure définitive qu'elle impose dans un débat où la prise en compte du local à travers l'enseignement de l'histoire et de la géographie sert à la fois de supplétif dans l'entreprise de construction du roman national et de substitut à la perte de substance linguistique en cours (Chanet 1996).

\footnotetext{
${ }^{8}$ https://www.persee.fr/doc/inrp_0000-0000_1995_ant_5_2_2076. Par la suite, il en sera fait mention sous la forme « document Persée ».
} 
En 1925, le ministre de l'Instruction va se trouver en quelque sorte contraint de préciser la doctrine de l'État à la suite de son interpellation par la Ligue pour la langue d'oc ${ }^{9}$ : celle-ci lui a en effet écrit afin qu'il autorise, par le biais d'une circulaire, le personnel de l'enseignement primaire à utiliser le dialecte comme adjuvant de l'enseignement du français. Dans son argumentaire elle mentionne entre autres l'existence de chaires universitaires ainsi que l'existence de cours de langue d'oc dans les écoles normales du Midi. L'ensemble du texte, habilement construit, débouche sur une demande somme toute modérée.

Le ministre a-t-il senti, derrière l'habileté rhétorique, le danger de la généralisation d'une telle revendication, une fois qu'elle aurait été satisfaite ponctuellement, ou bien a-t-il jugé qu'il convenait de sortir définitivement d'une ambiguité qui n'avait que trop duré ? L'un et l'autre sans doute, mais le ton adopté, l'argumentaire développé et la construction formelle du texte attestent la conviction qui conduit désormais à l'inéluctable généralisation du phénomène de minoration linguistique dans les régions à forte personnalité culturelle. Sans modifier subitement une réalité langagière encore vivace (une réalité sociologique ne peut évoluer, généralement, qu'avec le temps), il contribue à conforter, voire à accélérer la déprise linguistique en cours, avec plus ou moins de rapidité, sur l'ensemble du territoire. Pour la première fois depuis les rapports Grégoire et Barère et de façon officielle, il explique une attitude et fonde des choix en déclinant des principes de justification.

\section{Des réponses point par point}

La circulaire ne se contente pas d'offrir une réponse point par point aux arguments de la Ligue; elle élargit le débat en divulguant et ordonnant un discours du refus qui procède de nécessités que seuls peuvent maitriser les hommes responsables des affaires publiques et surtout elle rend compte ouvertement et synthétiquement d'une très longue tradition politico-culturelle de l'État qu'elle met pour la première fois en forme depuis 1794.

D'emblée, Anatole de Monzie écarte clairement la revendication posée par la Ligue : il n'est pas question d'accorder le moindre espace à l'enseignement des idiomes locaux dans l'enseignement primaire. La brèche ouverte au cœur de l'enseignement secondaire et supérieur dans les académies citées dans la circulaire doit être prestement colmatée pour éviter la double contagion qui gagnerait à la fois le primaire et l'ensemble des académies, dans un contexte politique global où les tenants de l'occitan à l'école tentent de récupérer le principe de la continuité des enseignements inscrit au programme du Cartel des gauches.

Le second argument du ministre concerne la pédagogie et donc directement le primaire. Pour lui, et malgré les discours de l'inspecteur général Bréal (Boutan 1998), il n'existe pas d'étayage savant à l'enseignement des dialectes. De plus, il prétend constater l'inexistence d'une pratique scolaire de terrain et le risque de balkanisation didactique ${ }^{10}$. Troisième étape, il souligne une contradiction de fond avec le discours didactique dominant dans l'enseignement des langues vivantes : «Comment, au surplus, accorder une telle proposition avec les méthodes générales de l'enseignement; avec la méthode directe dont il est usé pour apprendre l'allemand ou l'anglais ? $\gg{ }^{11}$. Quatrième étape, il rappelle le principe canonique défini par Jules Ferry et ses collaborateurs, l'enseignement du français par le français ${ }^{12}$. Cinquième et dernière étape, il met en exergue le dangereux couplage du risque psycho-linguistique et du risque didactique ; le

\footnotetext{
${ }^{9}$ Désormais, la Ligue.

10 «Qui songe à mettre en œuvre le breton, le basque ou le flamand pour faciliter l'intelligence du français ? Faudra-t-il édicter des règles différentes selon les régions et classer les idiomes d'après les services qu'ils seraient susceptibles de rendre à la pédagogie primaire? », document Persée, p. 335.

${ }^{11}$ Ibid.

12 «Se servira-t-on du languedocien comme truchement du français, tandis que le mot d'ordre moderne est l'apprentissage du français par le français? Les objectifs valables se multiplient, sans qu'apparaisse en sens contraire aucun argument décisif », ibid.
} 
recours systématique à un double signifiant pour un même signifié contient en germe les périls de paresse et de confusion intellectuelles, dans une époque où le bilinguisme est considéré comme cognitivement nocif et source de déloyauté patriotique (Tabouret-Keller 2011).

\section{Des réponses complémentaires}

Le ministre va enfin opposer l'irrecevabilité des langues locales en tant que langues de haute culture et de civilisation : « Il n'est pas indifférent à la formation de nos maitres d'apprendre n'importe quelle langue étrangère. Le maitre qui aura appris le français et le basque aura-t-il autant élargi ses capacités humaines et sociales que celui qui saura le français et l'allemand, le français et l'anglais, le français et l'espagnol ou l'italien, ou le russe ou toute autre langue étrangère riche de toute la vie intellectuelle et sentimentale d'un grand peuple moderne, expression de toute son activité, et de toute sa prospérité économique ? ${ }^{13}$. On perçoit ici les relents de l'évolutionnisme, idéologie qui opère un classement des civilisations selon leur degré d'intégration dans la modernité économique et technique du temps. De Monzie abonde en fait l'accusation possible de mépris, à tout le moins de condescendance, indéfectiblement attaché au locuteur d'une langue minorée.

De façon complémentaire, le ministre confortera son discours par quelques arguments totémiques :

- l'invocation de l'unité nationale pour laquelle, au bout du compte, la moindre allusion à la diversité dans l'unité semble toujours annoncer de lourdes menaces de décomposition ${ }^{14}$;

- le rappel de la longue croisade culturelle intérieure accomplie par les souverains et par les clercs, exhumant la tradition centralisatrice de la royauté ${ }^{15}$;

- la mise à nu, le fait est sans doute inédit dans les proportions que prend l'argument, des intentions des républicains : l'imposition du français passe sinon par l'éradication du moins par le confinement des dialectes ${ }^{16}$; on débouche donc à la fois sur l'affirmation tautologique de la valeur du français, langue de civilisation, témoin mythifié des combats du Peuple contre la royauté, contre l'hégémonisme linguistique du latin et contre le pouvoir de l'Église, et sur la cristallisation de la supériorité ontologique de la langue de la Nation sur l'ensemble de celles des petites patries;

- la mention de l'urgence de l'alphabétisation du peuple, dans un contexte de choix budgétaires contraints ${ }^{17}$; l'unification de la France demeurant à ses yeux inachevée (ce qui ne manque pas d'interpeler après la boucherie de 14-18, où tant de soldats qui ne parlaient pas ou très mal le français versèrent leur sang pour la patrie) il faut donc procéder aux meilleurs choix stratégiques.

\footnotetext{
${ }^{13}$ Document Persée, ibid.

14 « Mais si j'envisage le problème en surplombant ces disputes de méthodes, j'ai le devoir de me demander s'il est bon d'encourager les divisions linguistiques... La France, si unie qu'elle soit, ne l'est jamais trop », document Persée, p. 336.

15 « Depuis le temps où François I ${ }^{\mathrm{er}}$ faisait en 1539 la monarchie des écritures, en ordonnant que toutes les pièces de justice et d'administration seraient écrites en français, tous nos gouvernements ont professé une commune et constante doctrine d'unification linguistique », ibid.

${ }^{16}$ «L'école laïque, pas plus que l'Eglise concordataire, ne saurait abriter des parlers concurrents d'une langue française dont le culte jaloux n'aura jamais assez d'autels », ibid.

17 «Il m'est permis de faire observer, en outre, qu'il reste encore trop d'illettrés parmi nous pour que nous puissions distraire en faveur des plus respectables des parlers régionaux ou locaux une portion de l'effort nécessaire où la propagation du bon français "celui-là seul est vraiment français du cœur à l'âme et de la tête aux pieds qui sait, parle et lit la langue française”. Jusqu'à ce que cette définition de Musset soit applicable à l'unanimité des citoyens adultes, l'enseignement des patois devra être considéré comme un luxe et je vous prie de croire que notre époque n'est guère favorable aux dépenses de luxe pour compte de la collectivité », ibid.
} 


\section{Dédoublement et diglossie}

Il y aurait tout lieu de s'intéresser à la personnalité complexe du ministre de Monzie. Originaire de la Gironde, il connaitra une très longue carrière politique, exerçant quasiment sans discontinuer mandats électifs ou fonctions ministérielles de 1909 à 1940. Mais il occupe également une place notable dans le paysage intellectuel français : fondateur de l'Encyclopédie française, dirigée par Lucien Febvre de 1935 à 1966, il s'intéresse particulièrement au folklore et favorise à ce sujet les rencontres entre sociologues et ethnologues. Mais cette sympathie patrimoniale, qui le pousse à initier une démarche scientifique pluridisciplinaire d'un intérêt évident, ne fait pas de cet intellectuel issu des terres du Sud-ouest, où l'usage du vernaculaire est pour l'instant encore largement répandu, un sympathisant de la cause linguistique occitane.

De Monzie est un radical, et ce parti se montre d'abord partagé quant à la question linguistique avant d'opter pour des positions très jacobines, notamment à propos de la question alsacienne. Alors qu'avant 1914 ses congrès ont adopté des résolutions décentralisatrices, Edouard Herriot, président du Conseil, met un terme en juin 1924 aux dispositions administratives et législatives particulières à l'Alsace-Lorraine. Celle-ci, en 1908, avait été dotée d'une constitution lui conférant un large statut d'autonomie dans le cadre allemand. En fait, il semble que son intégration au sommet de l'État l'ait conduit à choisir entre un régionalisme à cultiver dans la confidence et un jacobinisme à défendre dans le cadre de ses convictions et de ses intérêts essentiels. On a affaire ici à l'illustration, dans les représentations d'un individu, de la répartition diglossique entre fonctions hautes et basses des langues dans le cadre d'un bilinguisme déséquilibré, c'est-à-dire un bilinguisme où une langue exerce son hégémonie sur l'autre. Mais il ne s'agit pas d'un phénomène isolé : confirmant l'ancrage social obligatoire de toute diglossie, d'autres ministres méridionaux, Georges Leygues, Maurice Faure, Edouard Daladier, cultiveront de façon plus ou moins nuancée l'amalgame ambigu de leur adhésion au félibrige et de positions jacobines assumées.

Quoi qu'il en soit, on a ici affaire à une poignée d'hommes d'État dont, en matière linguistique, l'action aura contribué à construire la cage et l'idéologie à y placer l'épervier, tout en désignant les idiomes qui vont endosser le rôle du passereau noir : « L'hypothèse est donc que la langue française s'instaure aussi sur le sacrifice. L'élimination des autres langues n'est pas une conséquence du succès du français mais sa condition. Le paradoxe est que l'ordre linguistique exige qu'il n'y ait qu'une langue mais s'accommode fort bien qu'il y ait une infinité de parlers. » (Sauzet $1988: 244-245$ ).

\section{L'embellie des parlers?}

On doit notamment à Philippe Martel (2005) une analyse fine et percutante de la progression des textes en faveur des langues régionales. Incontestablement, le tournant positif intervient après-guerre, en 1951, avec la loi Deixonne, lorsque l'on commence à réaliser que tous les tenants du régionalisme n'ont pas été des collaborateurs impliqués ou dilettantes. On connait l'infortune que connurent alors l'alsacien, le corse et le flamand, lesquels entraient encore dans le découpage territorial tracé par Barère en 1794 (les langues de l'étranger, donc celles des ennemis de la République). Le contenu de la loi, dont la circulaire d'application, texte indispensable pour la rendre opérante, ne fut publié qu'en 1969, laisse à penser que la représentation nationale tente de faire une place très modeste à des langues qu'elle ne considère au fond que sous la forme de parlers populaires et de témoignages d'un passé local dont il faut préserver la flamme vacillante. De plus, on est encore et toujours dans la dimension de l'adjuvant à l'enseignement du français, ainsi que le précise l'article 2: «Des instructions pédagogiques seront adressées aux recteurs en vue d'autoriser les maitres à recourir aux parlers 
locaux dans les écoles primaires et maternelles chaque fois qu'ils pourront en tirer profit pour leur enseignement, notamment pour l'étude de la langue française ».

Mais revenons à la chronologie martelienne, instituée en trois étapes :

- «le temps du refus fleuri » (p. 301) : c'est celui de la circulaire de Monzie et des années qui suivent ;

- «le temps des concessions maussades» (p. 305) : avec Deixonne, on retrouve la même hiérarchisation des idiomes que celle qu'avait établi de Monzie; il suffit pour s'en convaincre de citer cette explication du rapporteur de la loi (p. 307):

En bref, nous sommes partis du principe suivant: la langue, la littérature, le folklore, l'histoire d'une région constituent une richesse dont nul n'a le droit de dépouiller les populations qui en sont dépositaires. Mais ce ne serait plus enrichir, ce serait appauvrir que de substituer, même partiellement, un idiome local aux prodigieuses ressources de notre langue nationale, qui s'est révélée et qui est universellement reconnue comme le véhicule d'une pensée capable de défier les frontières et le temps. C'est pourquoi, sauf dans l'enseignement supérieur, où l'étudiant ne risque plus de désapprendre le français, nous avons localisé dans les activités dirigées les cours de langue locale. (...). Le même principe, toujours enrichir, ne jamais appauvrir, nous interdit de remplacer, au baccalauréat, les épreuves actuelles dans une grande langue étrangère, par des épreuves portant sur une langue régionale (...).

- «Le temps du verrouillage "citoyen" » (p. 310) : après la victoire de la gauche en 1981, Henri Giordan a remis un rapport au ministre de la culture ${ }^{18}$, dans lequel il propose de distinguer désormais la citoyenneté culturelle de la citoyenneté politique, ceci afin de développer une politique plus favorable à la pluralité linguistique de la France. Le 21 avril 1982, à l'Assemblée nationale, Michel Debré accuse Jack Lang de défaire l'unité nationale. Celui-ci se dérobe quant au fond, lui opposant le principe à ses yeux novateur de la décentralisation. On retrouve chez Debré des leitmotivs bien identifiés : lien entre langue et Nation, patrimonialisation (le mot n'est pas encore entré dans le champ politique) des langues par assimilation de celles-ci aux vieilles pierres, libre choix des individus et des familles et enfin, air nouveau, « la question de la citoyenneté et du communautarisme va se retrouver désormais au cœur des rares débats publics concernant les langues régionales » (p. 311). Les conséquences de ce raidissement se retrouveront dans le refus de ratification de la Charte européenne des langues ainsi que dans le celui de l'intégration de Diwan, en 2002. Il est vrai que l'article 2 de la Constitution, «la langue de la République est le français », voté en 1992 pour contrer la progression de l'anglais dans l'activité économique, deviendra une machine de guerre avec les contentieux déclenchés, notamment par les préfets, devant les tribunaux administratifs, lesquels constitueront au fil des années une véritable ligne Maginot jurisprudentielle. Aucun texte législatif protecteur, in fine, n'a jamais été voté, à l'exception de l'article 75-1 de la Constitution, dont la valeur et l'apport peuvent être en définitive considérés comme ectoplasmiques.

Il faut clore à présent ce tour d'horizon avec le vote de l'article L 312-10 du code de l'éducation, dans le cadre de la «loi n' 2013-595 du 8 juillet 2013 d'orientation et de programmation pour la refondation de l'école de la République », à l'initiative du ministre Vincent Peillon : «Les langues et cultures régionales appartenant au patrimoine de la France, leur enseignement est favorisé prioritairement dans les régions où elles sont en usage. Cet

\footnotetext{
${ }^{18}$ Démocratie culturelle et droit à la différence : rapport présenté à Jack Lang, ministre de la Culture, Paris, La
} Documentation française, 1982. 
enseignement peut être dispensé tout au long de la scolarité selon des modalités définies par voie de convention entre l'État et les collectivités territoriales où ces langues sont en usage... » Au départ, la rédaction du texte législatif se présentait de façon plutôt défavorable, on avait malencontreusement «oublié » les langues régionales. À l'initiative des militants culturels, occitans particulièrement, un travail substantiel fut effectué avec les services du ministère, lequel déboucha sur une insertion dans le corps de la loi dont on peut considérer qu'elle offrait au final non seulement un minimum de garanties et d'apaisements mais aussi un cadre un peu plus sécurisant.

\section{Langues régionales, langues de France : « une impossible laïcité linguistique »?}

Les parlers auxquels s'intéresse la loi Deixonne vont devenir les "langues régionales » à partir de 1976, sous la houlette du ministre de l'Éducation, René Haby. Si l'utilisation exclusive du terme « langue » constitue un progrès vis-à-vis de l'appellation « langues et dialectes » de la loi de 1951, la stabilité sémantique de l'expression prête à discussion selon Viaut et Pascaud (2017) : l'examen du contexte international le démontre à l'envi, on n'en a pas la même conception en France, où « régional » permet d'éviter l'emploi de « minoritaire », en Espagne, où l'on a affaire à des " langues propres », ou bien encore en Ukraine, où le terme "région » se voit « défini comme un terme générique désignant toute unité administrative autogérée qui peut aller de la République autonome de Crimée à une localité de taille modeste » (p. 5).

Dans l'optique de la ratification de la Charte européenne des langues régionales ou minoritaires $^{19}$, le rapport Cerquiglini, remis en 1999 au $1^{\text {er }}$ Ministre, Lionel Jospin, propose un recensement scientifique de 75 «langues de la France », ajoutant aux idiomes historiques de l'Hexagone les langues d'Outre-mer et un certain nombre de langues non territorialisées, en particulier celles de l'immigration. Du point de vue sociolinguistique, cette approche parait difficilement contestable : elle rend compte du multilinguisme effectif du pays, de sa richesse et de sa diversité. Cependant, le rapport prend ses distances avec l'optique adoptée par la Charte, basée sur une approche spatio-temporelle et donc, pour la France, sur le principe de "subordination géographique et d'antériorité chronologique par rapport à la diffusion concurrentielle du français standard » (Viaut et Pascaud $2017: 5$ ) :

Ce désir d'une assise géographique des langues régionales n'est pas sans contradiction avec l'intention culturelle que la Charte affiche. On peut faire valoir que la territorialisation systématique, issue du romantisme allemand qui inspira la linguistique du XIX $X^{e}$ siècle, s'oppose en outre :

- aux principes républicains français, qui tiennent que la langue, élément culturel, appartient au patrimoine national; le corse n'est pas propriété de la région de Corse, mais de la Nation.

- à la science, qui comprend mal l'expression " territoire d'une langue ». Ceci ne peut désigner la zone dont la langue est issue: en remontant le cours de l'histoire, on constate que toutes les langues parlées en France ont une origine "étrangère", - y compris le français, qui fut d'abord un créole de latin parlé importé en Gaule. La seule justification scientifique est d'ordre statistique, et de peu d'intérêt : elle revient à distinguer la zone qui, à l'heure actuelle, connait le plus de locuteurs d'un parler donné. En d'autres termes, le vrai territoire d'une langue est le cerveau de ceux qui la parlent.

\footnotetext{
${ }^{19}$ Désormais, la Charte.
} 
- à la réalité sociolinguistique, qui rappelle que la mobilité sociale contemporaine est telle que l'on parle les différentes langues "régionales" un peu partout. Le créole est une réalité linguistique bien vivante de la région parisienne. (Cerquiglini 1999 : 4-5)

Examinons donc l'argumentation ci-exposée, en suivant l'ordre dans lequel elle se déploie : - Les «principes républicains français » dont il est question ici relèvent du dogme de la langue exclusive et excluante de la Nation, tels qu'ils ont été posés par l'abbé Grégoire, portés par le Directoire et l'Empire, implémentés par la $\mathrm{III}^{\mathrm{e}}$ République, intimés par le ministre de Monzie dans le domaine de l'enseignement puis verrouillés, in fine, par l'article 2 de la Constitution, avec le renfort d'une jurisprudence adventice. Le 13 février 2020, à l'Assemblée nationale, le député Paul Molac présentait son projet de loi sur les langues régionales. S'étant livré à une critique de la conception « unilinguiste » (Lagarde 2019 : 15) qui prévalait en France, le député s'est fait reprendre par le ministre de l'Éducation car, en substance, on ne critique pas la République ${ }^{20}$. Si dans l'échange politique la posture joue un rôle important et, pour ainsi dire, conforme aux usages du débat démocratique, faut-il pour autant l'adopter au plan scientifique, par exemple en sous-entendant qu'en France on ne pourrait pas remettre en question, au moins au plan théorique, le principe abstrait de déspatialisation de la langue ? Daniel Baggioni (1986) a exposé avec justesse et profondeur la différence entre les conceptions allemande et française des concepts de langue et de peuple : si, en France, le débat a été tranché par l'Histoire sur le plan politique, aucune limite ne s'impose dans le champ scientifique. Tranché ? Voire, car l'article $1^{\mathrm{er}} \mathrm{du}$ statut de la Corse de 1991 mentionnait l'existence du «peuple corse, composante du peuple français ». Et si le Conseil constitutionnel a retoqué le texte en vertu du fait que l'on ne peut reconnaitre de sections au sein du Peuple, il n'a pas émis de véto lorsqu'il s'est agi de valider le principe de l'égal accès des femmes aux mandats électoraux avec la loi du 6 juin 2000. Certains juristes et hommes politiques avaient pourtant fait remarquer que l'on venait de distinguer une section au sein du Peuple et que l'on portait ainsi atteinte au principe constitutionnel d'indivisibilité de la souveraineté (Bereni et Lépinard 2004). Et si donc « le corse n'est pas propriété de la région de Corse, mais de la Nation », il ne faut pas s'étonner que le vote de la coofficialité le 17 mai 2013, par l'Assemblée de Corse, alors dirigée par une majorité de gauche, ait pu être écarté sans état d'âme par le gouvernement Valls, puisque le corse est propriété de la Nation, c'est-à-dire de l'État.

- Une langue n'aurait pas de territoire, si ce n'est « le cerveau de ceux qui la parlent» (sic). Cela semblerait signifier qu'elle ne peut dépasser les limites de la physiologie et du for intérieur de l'individu. Or cela heurte l'expérience que tout un chacun a de celle-ci, en tout cas de sa(ses) langue(s) de première acquisition, au sein de son foyer parental. Étant donné que l'homme est par nature un "parlêtre », selon le mot de Lacan, donc fondamentalement une entité vivante de langage, c'est par celui-ci qu'il se socialise : «...la langue, jamais innée, toujours acquise, irrigue toute construction sociale, de la cellule familiale à la communauté politique » (Arlettaz 2019 : 19). La réalité langagière dans sa totalité tend à démontrer que la langue n'a pas pour vertu principale de tourner dans le circuit fermé de l'intimité individuelle mais bien de mettre une personne en relation et en interrelation avec autrui. C'est par elle(s) que l'enfant se construit en s'identifiant non seulement aux siens mais aussi à elle(s) (Tabouret-Keller 1987), c'est par son truchement que s'exerce la réalité des rapports de pouvoir au sein de la société (Bourdieu 2014). Cette réalité-là doit d'ailleurs demeurer inaccessible aux consciences pour perdurer : « En tant que producteur de normes, l’État a nécessairement une langue ; cette impossible laïcité linguistique de l'État emporte

\footnotetext{
${ }^{20} \mathrm{http}$ ://videos.assemblee-nationale.fr/video.8757416 5e455466784c2.2eme-seance--mesures-de-justice-socialesuite--fonds-de-garantie-des-victimes-des-actes-de-terro-13-fevrier-2020.
} 
son impossible neutralité. Dans ce contexte, les langues rencontrent par nature le Pouvoir, qu'elles bénéficient dans l'État plurilingue du statut d'officialité - et donc de langues du Pouvoir - ou qu'elles viennent concurrencer dans l'État unilingue, la langue officielle - la langue du Pouvoir» (Arlettaz 2019: 21). Au total la conception désincarnée, quasi fictionnelle de la langue semble faire écho à une sorte de jusqu'auboutisme de la conception individualiste, dans une interprétation a maxima de la Déclaration des droits de l'Homme de 1789.

- Enfin la « réalité sociolinguistique », c'est bien sûr " la mobilité sociale contemporaine ». Si l'on ne peut nier la vigueur de ce fait social, faut-il pour autant en oublier, à tout le moins, les communautés humaines parlantes, qui partagent une conscience d'elles-mêmes à travers des comportements, des usages, des croyances, des valeurs et des langues ? Invoquer des communautés linguistiques comme a pu le faire Geneviève Vermes (1988) ferait-il donc courir un risque schismatique dans la France du XXI siècle ? Car si la communauté au sein de la Nation relève de la section du Peuple, «...toute reconnaissance de "catégories" ou mise en œuvre de "quotas" est présumée relever d'un modèle politique étranger fantasmé, celui du "communautarisme américain", et prend le risque d'ouvrir, selon l'expression consacrée, la "boîte de Pandore" des revendications particularistes » (Bereni et Lépinard 2004 : 80-81). Enfin, s'il est vrai que l'on peut entendre parler créole en région parisienne, il est tout aussi indéniable que les langues régionales ne sont par exemple enseignées, à de rarissimes donc improbables exceptions près, que dans les territoires historiques où elles se déploient, et qu'en définitive elles connaissent une forme d'assignation spatiale tout à fait contradictoire avec l'énoncé des principes avancés dans le rapport.

\section{Une dernière investigation dans le champ théorique}

Une fois n'est pas coutume, et puisque nous avons abordé la question de l'individualisme, nous effectuerons un dernier détour par la philosophie en prêtant attention aux travaux de Vincent Descombes, qui s'est intéressé aux concepts de sujet, de subjectivité, d'individu et d'identité. Cet auteur tente de répondre au sentiment de perte de densité et de substance sociales engendré par l'individualisme en prenant soin de distinguer, en tant que fondement du sujet « la liberté comme indépendance et la liberté comme autonomie »(2004:333). Si l'on peut basculer, avec la première, dans une forme d'anomie dont Tocqueville craignait qu'elle ne fût la conséquence ultime de la démocratie et le ferment de sa destruction (Allemand 2000), la visée d'autonomie «n'est justement pas le fait d'un individu qui se met à part, mais bien d'un sujet qui ne se conçoit qu'en relation de communication intersubjective avec d'autres sujets » (ibid.), soit dans une relation holiste, donc de subordination, de la partie au tout. En conséquence de quoi «la liberté véritable n'est pas dans la liberté naturelle mais dans "la soumission à des règles librement acceptées" (p. 335). Donc, dans la relation hiérarchique consentie entre l'individu et société placée au-dessus de lui, Descombes, ayant le souci de fonder philosophiquement une théorie de l'agir social, propose une articulation de ses spéculations avec l'appréhension du réel, par exemple dans le domaine de l'identité groupale. Et il nous faut le citer ici longuement car sa démonstration nécessite des développements importants. Interrogé par la revue Esprit $(2000)^{21}$, voici ce qu'il répond à la question suivante : «Comment penser de concert les valeurs individualistes contemporaines et la notion de hiérarchie ?» (p. 158).

21 https://esprit.presse.fr/article/descombes-vincent/vers-une-anthropologie-comparative-des-democratiesmodernes-entretien-ii-9294. 
On oppose volontiers des "républicains » soucieux de l'universel, de l'égalité devant la loi voulue par les citoyens, et des "libéraux" ou "libertaires " qui comprennent les droits de l'homme comme des protections accordées à l'individu contre l'État, et donc aussi contre la Loi républicaine quand elle n'est en fait que la volonté d'une majorité....

Ces débats ont en commun de mettre en scène des demandes de reconnaissance émanant de groupes qui s'estiment mal représentés dans le cadre du modèle intégrateur de la République française tel qu'il a existé jusqu'à nous. Il faut, diton, reconnaitre l'autre... (p. 161)

Ce type de demande correspondrait par exemple, de son point de vue, au mariage pour tous, à une réponse positive aux aspirations des groupes dits LGBT ou encore aux revendications régionalistes.

Les revendications égalitaires expriment une demande de reconnaissance équistatutaire, elles réclament la fin d'une discrimination, l'instauration d'une règle d'indifférenciation. (ibid.)

Tel serait par exemple le cas de la parité homme-femme ou encore la lutte pour les droits civiques des noirs menée aux États-Unis par Martin Luther King.

Les revendications identitaires expriment au contraire la demande d'une reconnaissance hiérarchique, puisqu'elles veulent un statut spécial. Or, l'actualité nous offre plusieurs exemples d'une confusion intellectuelle entre ces deux types de revendication. Nous constatons que la demande d'une reconnaissance hiérarchique y est présentée dans l'idiome de la reconnaissance équistatutaire (celui des droits de l'homme, des prétentions de l'individu, de la défense des minorités contre la tyrannie majoritaire). Autrement dit, ces revendications mettent en jeu des représentations hybrides, des représentations dans lesquelles le principe holiste n'est pas déclaré comme tel, mais cherche à se faire passer pour un principe égalitaire. (ibid.)

Soit parexemple la question de la reconnaissance d'autres langues que la langue nationale comme langues légitimement utilisables dans certains actes publics. Lorsqu'un Breton se soucie de la langue bretonne, qu'un jeune issu de l'immigration berbère se soucie de la langue berbère, ce ne sont pas des individus qui réclament un droit individuel à s'exprimer dans une langue de leur choix. En fait, derrière la rhétorique des droits de l'homme, on doit entendre la voix d'un "homme collectif», d'un être (lui nous parle, comme le font toutes les sociétés traditionnelles, du devoir de transmettre à ses petits-neveux la langue et la culture ("l'identité ») qu'on se représente comme son mode d'intégration au monde et à l'histoire : si notre langue devait disparaitre, pensent-ils, ce serait comme si nous n'avions pas existé ou pas mérité d'exister. (ibid.)

On peut lire ici une façon lucide et rigoureuse de poser le problème du lien entre langue et communauté parlante, ou encore entre langue et identité. En affirmant qu'une revendication linguistique suppose par nature son adossement à un collectif, Descombes reprend le principe holiste selon lequel il ne peut y avoir d'individu sans société et vice-versa (Elias 1991).

Dans la controverse publique, ceux qui incarnent le courant " jacobin » ont raison de noter que la revendication d'un droit à la différence contredit le principe républicain, et donc la définition même du citoyen français. Et, en effet, il n'y a pas 
de compromis possible sur le principe. Mais ils ont tort de croire qu'une société puisse vivre selon les normes d'un universalisme abstrait. (ibid.)

Et c'est bien l'adossement, nous semble-t-il, proposé dans le rapport Cerquiglini, puisque toute relation entre un locuteur et la communauté des parlants y est occultée au nom du principe philosophique de la primauté de l'individu souverain. Si l'on peut considérer que l'auteur du texte a raison sur un plan abstrait, la réalité des choses le conduit à une impasse intellectuelle.

Leur erreur est de s'imaginer que le tout de la vie sociale puisse être intégralement représenté dans le seul domaine politique, celui où des sujets rationnels décident ensemble des conditions de leur association. Déjà... les républicains français ont mis longtemps avant d'admettre qu'il pouvait y avoir des revendications collectives, pas seulement individuelles. Sur le plan du pur principe idéologique, il n'aurait dîu en effet y avoir que des revendications présentées à chaque fois par des citoyens particuliers qui s'estimaient lésés dans leurs intérêts personnels. Or, la formation de syndicats ouvriers heurte ce principe : voici des groupes particuliers qui prétendent parler au nom d'un intérêt général distinct, autrement dit d'une valeur (puisqu'un syndicat ouvrier va tendre à défendre, non pas seulement ses membres, mais le métier, ceux qui en vivent aujourd'hui et ceux qui en vivront demain). (p. 162)

Sur le sujet particulier des syndicats, il semble que l'actuel gouvernement veuille revenir aux sources du républicanisme, à une forme de pureté idéologique originelle puisqu'il a fait voter le 6 août 2019 par l'Assemblée nationale la loi de transformation de la fonction publique. Subséquemment, par décret du $1^{\text {er }}$ décembre 2019, au sein de celle-ci, les commissions administratives paritaires ne sont plus compétentes pour examiner les décisions individuelles en matière de mobilité applicables à compter du 1er janvier 2020, ainsi que pour les décisions individuelles en matière de promotion applicables à partir du $1^{\mathrm{er}}$ janvier 2021. En oblitérant la médiation syndicale dans le déroulé des carrières des fonctionnaires, ces derniers se retrouvent donc dans la situation de négocier seuls leurs intérêts de carrière face à l'administration...

Il en va de même des revendications dites "communautaires". Ici encore, le républicain strict se heurte à une demande qui ne correspond à rien de légitime dans son système de pensée. Dans un mouvement régionaliste ou communautariste, il ne peut voir qu'une association particulière qui se voudrait investie de la charge de défendre une "identité », c'est-à-dire une valeur transcendant la simple particularité des individus, sans pourtant être investie d'en haut par la puissance publique incarnant la souveraineté nationale. Il manque donc au "jacobin» la sagesse d'admettre que le principe de l'assimilation (en elle-même nécessaire à long terme) doive parfois accepter de composer avec une réalité qui lui résiste. (ibid.)

Descombes appelle donc à une certaine modération dogmatique en vertu du principe de réalité. Les choses étant ce qu'elles sont, on doit transiger pour parvenir à un but que l'on sait ne pouvoir atteindre dans l'immédiat mais dont on a conscience qu'il constitue bien l'objectif terminal de son action. Ce qui signifie, si l'on comprend son propos, qu'il faut accepter d'en passer par des situations de compromis dans un souci de maintien de la concorde sociale et politique.

Nous ferons ici, pour en terminer, trois remarques : la première, c'est que Descombes met en exergue les pièges d'une pensée par trop rationaliste, trop radicale et, réciproquement, de son point de vue tout au moins, ceux de la confusion qui peut s'installer dans certaines revendications dont le positionnement conceptuel lui apparait confus, hésitant et flottant, en 
tout cas mal fondé en logique. La deuxième, c'est que l'universalisme dont il se réclame, celui de la Déclaration des droits de l'Homme, demeure malgré tout un universalisme centré, lequel ne correspond ni plus ni moins qu'à la conception française de la souveraineté :

Quand une société est ainsi dressée à se représenter l'homme dégagé de toutes les contingences nationales et historiques, dans ce qu'il a de plus général et de plus abstrait, elle ne peut s'attacher qu'à un idéal qui lui paraisse valable pour le genre humain tout entier. De ce point de vue, par conséquent le Français ne peut, sans contredire sa mentalité, se poser les problèmes moraux ou politiques dans des termes étroitement nationaux. Quand il légifère, c'est pour l'humanité entière qu'il croit légiférer, puisque l'humanité est la seule réalité véritable, et que les formes superficielles dans lesquelles elle s'enveloppe et qui la particularisent aux différents moments de l'histoire ne permettent pas de plus de retenir l'attention du philosophe et de l'homme d'État que du poète. (Durkheim 1938 : 314).

Et il nous semble pertinent d'affirmer que le positionnement de Descombes fait référence à ce « cosmopolitisme constitutionnel» (ibid.) dont parle Durkheim. Mais ce faisant et pour la question qui nous occupe, Descombes a en quelque sorte le choix entre la stratégie révolutionnaire de Grégoire, l'éradication pure et simple des patois, et celle de Barère, qui appelle à la modération et à la transaction même si, au final, le dessein est le même. Et, sans commettre d'anachronisme, il nous semble déceler dans son discours une référence potentielle à Barère plutôt qu'à Grégoire. Troisième et dernière remarque, c'est que l'universalisme n'est pas un, que la citoyenneté française n'est pas LA citoyenneté tout court, qu'il n'existe en l'occurrence aucun empyrée de la raison parce qu'aucun universalisme ne peut s'auto-décreter libre de toute accroche spatio-temporelle ou de tout déterminisme.

Du rapport Cerquiglini aux travaux de Descombes se donnent donc à lire deux postures entre lesquelles existent plus que des nuances mais dont l'une paraît beaucoup plus fouillée que l'autre. Cependant il faut bien considérer que le rapport a été rédigé dans un temps très court, en direction des responsables en charge des destinées du pays, dans un contexte où les filiations idéologiques pesaient évidemment de tout leur poids, de quelque côté que l'on se trouve de l'échiquier politique, particulièrement en période de cohabitation ${ }^{22}$. Le temps du politique, ses contraintes, ses contingences, ne concerne évidemment pas celui du clerc, tout entier dans son grand-œuvre par le truchement et la grâce de la skholè.

Pourtant, si l'on revient une dernière fois sur la question de l'universalisme, un autre chemin y conduisant est par exemple proposé par la Déclaration universelle des droits linguistiques ${ }^{23}$, signée à Barcelone, en juin 1996, laquelle s'inspire du principe de souveraineté de toute communauté humaine consciente d'elle-même en regard de toutes les autres et non point contre toutes les autres ou encore contre au moins l'une d'entre elles : « La présente Déclaration prend donc, de ce fait, comme point de départ les communautés linguistiques et non pas les Etats. Elle s'inscrit dans le cadre du renforcement des institutions internationales capables de garantir un développement durable et équitable pour toute l'humanité, tout en poursuivant l'objectif de favoriser l'organisation d'un cadre politique de la diversité linguistique fondé sur le respect mutuel, la cohabitation harmonieuse et la défense de l'intérêt général » (p. 23).

\footnotetext{
${ }^{22}$ Nous vivons alors la troisième cohabitation, qui va de 1997 à 2002 et concerne Jacques Chirac, Président de la république, et Lionel Jospin, $1^{\text {er }}$ Ministre. Elle a débuté deux ans après le début du septennat et se poursuit jusqu'à la fin du mandat présidentiel.

${ }^{23} \mathrm{http}$ ///www.axl.cefan.ulaval.ca/Langues/Declaration_univ-droits_ling1996.htm.
} 


\section{Conclusion}

On aura compris que ce texte vise à mettre au clair les conséquences d'une réforme, celle du baccalauréat, susceptible de remettre en cause l'existence et la pérennité des langues régionales dans l'enseignement, seul espace de vie qui leur ait été réellement concédé. Ce qui est en question ici n'est rien moins que le risque difficilement réfutable de leur déclin et de leur consomption. L'hypothèse émise par Patrick Sauzet (1988), celle d'un choix sacrificiel de toutes les langues du territoire au bénéfice d'une seule, semble donc constituer un scénario crédible dans un avenir relativement proche. Crédible ne veut cependant pas dire irréversible, certains résultats tout à fait tangibles ont été obtenus dans le temps long : ils ont, d'une part, permis l'institutionnalisation d'un enseignement qui répondait à une demande avérée, d'autre part engendré la structuration progressive d'une didactique correspondant aux canons scolaires du temps, grâce à la constitution et à la professionnalisation d'un corps d'enseignants. De plus, il n'est pas dit que les collectivités territoriales, plusieurs ont mis en place des offices de la langue, la Collectivité de Corse affiche un programme ambitieux, n'aient pas à l'avenir leur mot à dire, en particulier dans l'avancée démocratique d'une décentralisation avérée et significative.

Il n'a cependant pas paru inutile à l'auteur de cette étude de tenter de mettre en évidence les jalons d'une pensée aussi tendue sur ses ergots que l'orgueilleux épervier toisant le frêle merle dans la cage qu'ils doivent partager. Si cette pensée procède du politique, donc de l'idéologie, elle trouve à se nourrir largement dans le domaine intellectue ${ }^{24}$, ici la sociolinguistique et la philosophie avec, on a pu le constater, tout un ensemble de nuances permettant de bien différencier les apports des deux auteurs ici étudiés, Cerquiglini et Descombes.

Au final, il reste à constater que la porte de la cage s'est refermée sur les deux protagonistes de la parabole initiale et à souhaiter que l'épervier, par nonchalance, désintérêt ou manque d'appétit, ne prête provisoirement qu'un oil distrait à son compagnon de captivité et d'infortune... À moins que, improbable et miraculeux accident, une main maladroite ne libérât involontairement les deux volatiles. Ou bien encore, osons l'imaginer, qu'elle choisit de délibérément de leur rendre leur liberté, dans la volonté affirmée d'apporter le souffle du renouveau dans l'espace politique et social.

\section{Bibliographie}

Allemand Sylvain, 2000, "Alexis de Tocqueville et l'exercice de la démocratie », in $L a$ sociologie Histoire et idées, Auxerre, Editions Sciences humaines, pp. 25-27.

Arlettaz Jordan, 2019, «Langue régionale, langue minoritaire : une terminologie porteuse de sens ? », in Les langues régionales et la construction de l'État en Europe, Actes du colloque organisé les 7 et 8 juin 2018 par l'IRDEIC-Centre d'Excellence Jean Monnet, Amane Gogorza et Wanda Mastor (dirs.), Paris, LGDJ, pp. 19-40.

Baggioni Daniel, 1998, «Préhistoire de la glottopolitique dans la linguistique européenne, de J. G. Herder au Cercle linguistique de Prague », Langages, n 83, 1986, pp. 35-51.

Boutan Pierre, 1998, De l'enseignement des langues : Michel Bréal linguiste et pédagogue, Paris, Hatier.

Bereni Laure, Lépinard Éléonore, 2004, « “Les femmes ne sont pas une catégorie” les stratégies de légitimation de la parité en France », Revue française de science politique, vol. 54(1), pp. 71-98.

\footnotetext{
${ }^{24}$ Nous préférons cet adjectif plutôt que le terme «scientifique », car la philosophie ne peut pas être considérée comme une science humaine stricto sensu (Giroux, Tremblay, 2002), quand bien même elle constitue, au plan général, un très puissant et très efficace outil au service de la formation des concepts.
} 
Bourdieu Pierre, 2014, Langage et pouvoir symbolique, Paris, Points.

Chanet Jean-François, 1996, L'école républicaine et les petites patries, Paris, Aubier.

Chervel André,1995, L'enseignement du français à l'école primaire Textes officiels, Tome 2 : 1880-1939, Paris, INRP Economica.

Calvet Louis-Jean, 1979, Linguistique et colonialisme, Paris, petite bibliothèque Payot.

Descombes Vincent, 2004, Le complément de sujet, Paris, nrf essais, Gallimard.

Durkheim Emile, 1999 [1938], L'évolution pédagogique en France, Paris Quadrige, PUF.

Elias Norbert, 1991, La société des individus, Paris, Fayard.

Girard René, 1972, La violence et le sacré, Paris, Grasset.

Lagarde Christian, 2019, «Ce que nous apprend la sociolinguistique : une langue régionale estelle une "autre" langue? », in Les langues régionales et la construction de l'État en Europe, Actes du colloque organisé les 7 et 8 juin 2018 par 1'IRDEIC-Centre d'Excellence Jean Monnet, Amane Gogorza et Wanda Mastor (dirs.), Paris, LGDJ, pp. 7-18.

Martel Philippe, 2005, Le "patois à l'école»? Retour sur un débat (XIXe-XXe siècles), in Marges linguistiques, $\mathrm{n}^{\circ} 10$, Saint-Chamas, M.L.M.S. éditeur, www.margeslinguistiques.com, pp. 301-317.

Ottavi Pascal, 2008, Le bilinguisme dans l'école de la République? Le cas de la Corse, Ajaccio, Albiana.

Sauzet Patrick, 1988, «L'occitan Langue immolée », in Vingt-cinq communautés linguistiques de France, Geneviève Vermes (dir.), Paris, L'Harmattan, pp. 208-260.

Tabouret-Keller Andrée, 1987, «Questions en vue d'une psychologie clinique du bilinguisme ", in France pays multilingue, Tome 2, Pratiques des langues en France, Josiane Boutet et Geneviève Vermes (dirs.), Paris, L'Harmattan, pp. 179-192.

Tabouret-Keller Andrée, 2011, Le bilinguisme en procès, cent ans d'errance (1840-1940), Limoges, Lambert Lucas.

Viaut Alain, Pascaud Antoine, 2017, « Pour une définition de la notion de "langue régionale" », Lengas, $\mathrm{n}^{\circ} 82$, http://journals.openedition.org/lengas/1380, consulté le 25 avril 2018. 


\section{GLOTTOPOL}

Revue de sociolinguistique en ligne

Comité de rédaction : Michaël Abecassis, Salih Akin, Sophie Babault, Claude Caitucoli, Véronique Castellotti, Régine Delamotte, Robert Fournier, Stéphanie Galligani, Emmanuelle Huver, Normand Labrie, Foued Laroussi, Benoit Leblanc, Fabienne Leconte, Gudrun Ledegen, Danièle Moore, Clara Mortamet, Alioune Ndao, Isabelle Pierozak, Gisèle Prignitz.

Rédactrice en chef : Clara Mortamet.

Comité scientifique : Claudine Bavoux, Michel Beniamino, Jacqueline Billiez, Philippe Blanchet, Pierre Bouchard, Ahmed Boukous, Pierre Dumont, Jean-Michel Eloy, Françoise Gadet, Monica Heller, Caroline Juilliard, Jean-Marie Klinkenberg, Jean Le Dû $(\dagger)$, Marinette Matthey, Jacques Maurais, Marie-Louise Moreau, Robert Nicolaï, Didier de Robillard, Paul Siblot, Claude Truchot, Daniel Véronique.

\section{Comité de lecture pour ce numéro :}

Salih Akin, Carmen Alén Garabato, Sophie Babault, Philippe Blanchet, Henri Boyer, Véronique Castellotti, Marisa Cavalli, Jean-François De Pietro, Didier de Robillard, Alain Di Meglio, Ksenija Djordjevic, Jean Michel Eloy, Pascale Erahrt, Véronique Fillol, Monica Heller, Robert Fournier, Normand Labrie, Hervé Lieutard, Jean Le Dû (†), Marinette Matthey.

http://glottopol.univ-rouen.fr

ISSN : 1769-7425 